\title{
Mechanical Structure Design and Robust Adaptive Integral Backstepping Cooperative Control of a New Lower Back Exoskeleton
}

\author{
Haoping WANG, Yujuan SUN, Yang TIAN* \\ Sino-French International Joint Laboratory of Automatic Control and Signal Processing (LaFCAS), \\ School of Automation, Nanjing University of Science \& Technology, Nanjing 210094, China \\ tianyang@njust.edu.cn (*Corresponding author)
}

\begin{abstract}
A new wearable power assist device for lower back support is proposed in this paper. The waist power assist suit is designed to relieve lower back pain and it is equipped with four pushrods to realize the three-dimensional rotation around the waist joint. The mathematical model of the device including the kinematics and the dynamics is presented on the basis of the freedom analysis. The model of the pushrod considering friction described in modified lugre model is given in the third part. A kind of lumbar rehabilitation movement is put forward. To ensure the coordination work of the four motors, an improved mean deviation coupling control strategy is proposed. Displacement tracking controller based on robust adaptive integral backstepping control is adopted to deal with friction. To demonstrate the effectiveness of the proposed synchronous control strategy, simulation is carried out in Matlab/Simulink and the simulation results show better performances when compared to deviation coupling cooperative control. At last, simulation has been carried out using the Anybody software in order to prove the availability of the mechanism.
\end{abstract}

Keywords: Lower back, Exoskeleton, Kinematics, Dynamics, Mean deviation coupling, Cooperative control.

\section{Introduction}

In recent years, lower back pain has become a common health problem that causes negative effects on people's daily life and is a leading cause of disability (Hagen \& hune, 1998). Yet lower back pain not only creates health risks but also economic losses, including the cost of medical care for treating and other related losses because of the decline in work efficiency (Maniadakis \& Gray, 2000).

So far, several researchers have developed wearable power-assist suits to assist human motion and reduce burden of the waist such as Smart suit lite (Imamura et al., 2014), PLAD (AbdoloEramaki et al., 2007), WSAD (Luo \& Yu, 2013) and AB-Wear (Inose, Mohri \& Yamada, 2017, Inose et al., 2017). Most of these exoskeletons are limited to the flexion and extension while few devices assist in the lateral flexion and torsion. Another limitation is that the existing lumbar exoskeletons need to be fixed to the shoulder or thigh, which will cause extra burden on the shoulder, or will bring about some inconvenience to walk.

This study proposes a new wearable power assist device for lower back support. As it is shown in Figure 1, just using a belt equipped with four pushrods, the suit is able to share the weight of the upper body so as to relieve lumbar pressure with no extra load on the other body part. The device can be considered as a 3-DOF(degrees of freedom) parallel mechanism. It can decompress the spine and assist waist motion in three rotational degrees of freedom by controlling the length of the four pushrods, which makes it essential for achieving the cooperative work of the four motors. In addition, considering the userfriendliness and security of the device, the slowerrunning tracking precision of the four motors deserves close attention. The inherent nonlinear and uncertain factors of the linear motor are the main reasons to influence the control accuracy. As it is widely known, for an electro-mechanical system with the characteristic of low speed, the control system performance is mainly affected by factors such as friction torque, motor fluctuations torque, measurement system error etc (Zhou et al., 2017). Among these factors, the friction torque is predominant over others, which directly affects the control accuracy and movement stability. Thus in this paper, nonlinear friction is taken into consideration due to its bad influence on the performance of high precision servo control system. In the existing specialized literature, a wide range of adaptive estimation techniques have been proposed for friction compensation (Wang et al., 2016, Ahmed et al., 2018). Most of them are based on a friction model, for example, on the simple Coulomb model, or on more sophisticated ones like the LuGre model (Canudas, Olsson \& Astrom, 1995) which is able to describe the friction effects more accurately and addresses low velocities and velocity reversals (Wang, Wang \& Chai, 2011). To study the dynamics of a 
system with friction and develop a controller for eliminating the frictional instability, an accurate friction model that can display a wide range of observed friction phenomena is needed. A new friction model is proposed in this paper based on the well known LuGre friction model that can accurately describe the nature of friction force with parameter uncertainties. To reduce the effect of disturbances and parameter variations, a robust adaptive integral backstepping control is adopted on the base of the relevant nonlinear observers.

The rest of this paper is organized as follows. Section II presents the mechanical structure and freedom analysis of the lower back exoskeleton. Section III gives the mathematical model of the device. In section IV, an improved mean deviation coupling control strategy is proposed to control the cooperation of the pushrods and the robust adaptive integral backstepping control is taken to control single motor. The practicality of the device is discussed in section V. Simulation results are shown in section VI. Finally, conclusions and perspective are shown in the seventh section.

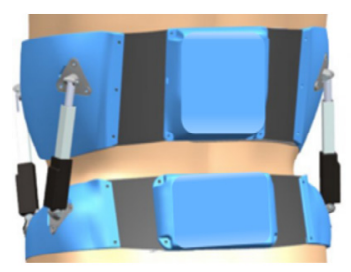

(a)

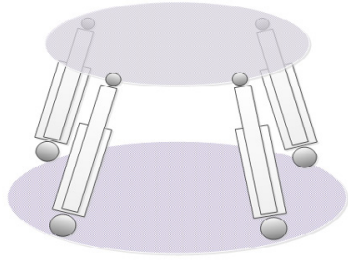

(b)
Figure 1. Structure design of lower back exoskeleton [(a) Mechanical appearance, (b)Simplified Structure]

\section{Exoskeleton Structure}

\subsection{Mechanical Structure}

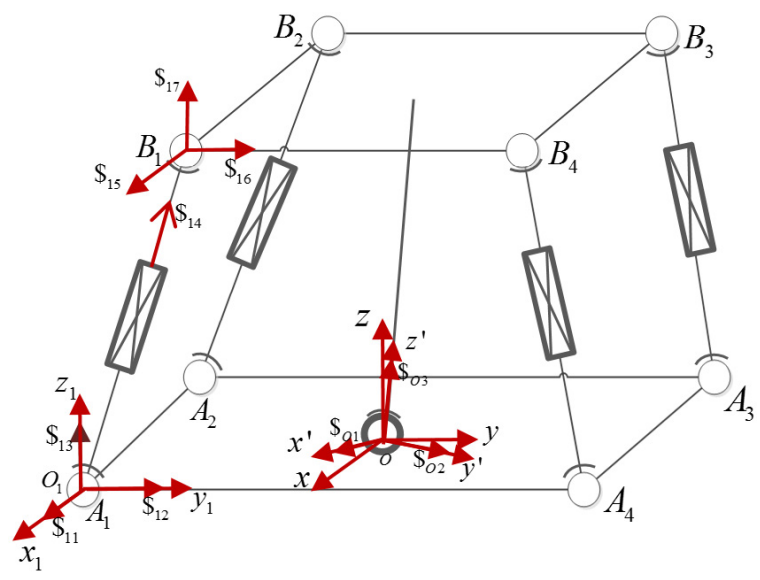

The proposed lower back exoskeleton can be considered as a parallel mechanism composed of a moving platform(waist platform), a fixed platform(pelvis platform) and four limbs connecting the two platforms. As it is shown in Figure 2, the both platforms can be considered rectangles. Each limb contains a prismatic joint and two spherical joints. The degree of freedom of the mechanism can be analyzed based on the screw theory.

\subsection{Freedom Analysis}

Setting up a coordinate system $o_{i}-x_{i} y_{i} z_{i}$ at point $A_{i}$, the screw system can be obtained (Kim, Jin \& Park, 2015)

$\$_{i 1}=\left(\begin{array}{lllllll}1 & 0 & 0 & ; & 0 & 0 & 0\end{array}\right)$

$\$_{i 2}=\left(\begin{array}{lllllll}0 & 1 & 0 & ; & 0 & 0 & 0\end{array}\right)$

$\$_{i 3}=\left(\begin{array}{lllllll}0 & 0 & 1 & ; & 0 & 0 & 0\end{array}\right)$

$\$_{i 4}=\left(\begin{array}{lllllll}0 & 0 & 0 & ; & p_{1} & m_{1} & n_{1}\end{array}\right)$

$\$_{i 5}=\left(\begin{array}{lllllll}1 & 0 & 0 & ; & 0 & m_{2} & n_{2}\end{array}\right)$

$\$_{i 6}=\left(\begin{array}{lllllll}0 & 1 & 0 & ; & p_{2} & 0 & n_{3}\end{array}\right)$

$\$_{i 7}=\left(\begin{array}{lllllll}0 & 0 & 1 & ; & p_{3} & m_{3} & 0\end{array}\right)$

with $p_{j}, m_{j}, n_{j}$ representing different real numbers. From (1), it can be noticed that the motion screw is full rank, which means that the four branches will not exert force constraints on the moving platform. When human body is taken into account, it can be regarded as a spherical joint and its screw system is

$\$_{O 1}=\left(\begin{array}{lllllll}1 & 0 & 0 & ; & 0 & 0 & 0\end{array}\right)$

$\$_{O 2}=\left(\begin{array}{lllllll}0 & 1 & 0 & ; & 0 & 0 & 0\end{array}\right)$

$\$_{O 3}=\left(\begin{array}{lllllll}0 & 0 & 1 & ; & 0 & 0 & 0\end{array}\right)$

Its reciprocal screw can be obtained as follows:

$\$_{O 1}^{T}=\left(\begin{array}{lllllll}1 & 0 & 0 & ; & 0 & 0 & 0\end{array}\right)$

$\$_{O 2}^{T}=\left(\begin{array}{lllllll}0 & 1 & 0 & ; & 0 & 0 & 0\end{array}\right)$

$\$_{O 3}^{T}=\left(\begin{array}{lllllll}0 & 0 & 1 & ; & 0 & 0 & 0\end{array}\right)$

The reciprocal screw is represented by the three force screws along the $\mathrm{x}, \mathrm{y}$ and $\mathrm{z}$ axes respectively, which restrict the movement of the moving platform along the corresponding axis.

According to the modified Grübler-Kutzbach criterion (Liu, Li \& Huang, 2011)

$M=d(n-g-1)+\sum_{i=1}^{g} f_{i}+v-\zeta=6(10-13-1)+31+0-4=3$

https://www.sic.ici.ro 
where $M$ denotes the DOF of the mechanism, $d$ is the mechanism order, $n$ is the number of links including frame, $g$ is the number of kinematic joints, $f_{i}$ is the DOFs of the $i$ th joint, $v$ is the number of parallel redundant constraints, $\zeta$ is the mechanism passive freedoms.

From equation (4), it can be observed that the moving platform can realize three-dimensional rotation around waist joint, that is, as shown in Figure 3, lateral flexion, rotation, flexion and extension.

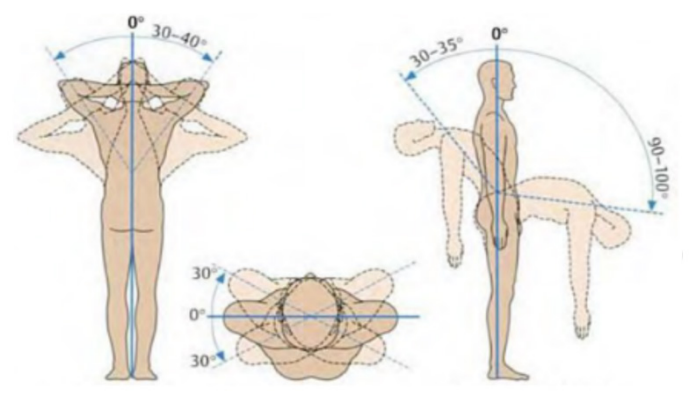

Figure 3. Waist movement

\section{Mathematical Model}

\subsection{Kinematic Model}

The mechanism can be simplified to the structure shown in Figure 4. The four prismatic joints of the limbs work as drivers and the input variables are defined as $l_{1}, l_{2}, l_{3}, l_{4}$. The output variables are the attitude angles of the moving platform denoted as $\gamma, \beta, \alpha$ (Hou, Zhao \& Fan, 2014). As shown in Figure 4, the fixed coordinate system $O-x y z$ and the moving coordinate system $O^{\prime}-x^{\prime} y^{\prime} z^{\prime}$ are set up at the spherical joint of the middle branch. The $\mathrm{x}$-axis of the fixed coordinate system is parallel to $A_{1} A_{2}$ and point to $A_{1}$ while x'-axis is parallel to $B_{1} B_{2}$ and point to $B_{1}$. The $\mathrm{Z}$-axis is perpendicular to the fixed platform while the $z^{\prime}$-axis is perpendicular and point to the moving platform.

The position vector of the spherical joint $B_{i}$ in the moving coordinate system is

$$
\begin{aligned}
B_{1}{ }^{\prime} & =\left[\begin{array}{lll}
\frac{1}{2} n_{B} & -\frac{1}{2} m_{B} & h
\end{array}\right] \\
B_{2}{ }^{\prime} & =\left[\begin{array}{lll}
-\frac{1}{2} n_{B} & -\frac{1}{2} m_{B} & h
\end{array}\right] \\
B_{3}{ }^{\prime} & =\left[\begin{array}{lll}
-\frac{1}{2} n_{B} & \frac{1}{2} m_{B} & h
\end{array}\right] \\
B_{4}{ }^{\prime} & =\left[\begin{array}{lll}
\frac{1}{2} n_{B} & \frac{1}{2} m_{B} & h
\end{array}\right]
\end{aligned}
$$

The position vector of the spherical joint $A_{i}$ in the fixed coordinate system is

$$
\begin{aligned}
& A_{1}=\left[\begin{array}{lll}
\frac{1}{2} n_{A} & -\frac{1}{2} m_{A} & 0
\end{array}\right] \\
& A_{2}=\left[\begin{array}{lll}
-\frac{1}{2} n_{A} & -\frac{1}{2} m_{A} & 0
\end{array}\right] \\
& A_{3}=\left[\begin{array}{lll}
-\frac{1}{2} n_{A} & \frac{1}{2} m_{A} & 0
\end{array}\right] \\
& A_{4}=\left[\begin{array}{lll}
\frac{1}{2} n_{A} & \frac{1}{2} m_{A} & 0
\end{array}\right]
\end{aligned}
$$

where $m_{B}$ is the length of $B_{1} B_{4}, n_{B}$ is the length of $B_{1} B_{2}, h$ is the distance between the center of the two platforms. $m_{A}$ is the length of $A_{1} A_{4}, n_{A}$ is the length of $A_{1} A_{2}$.

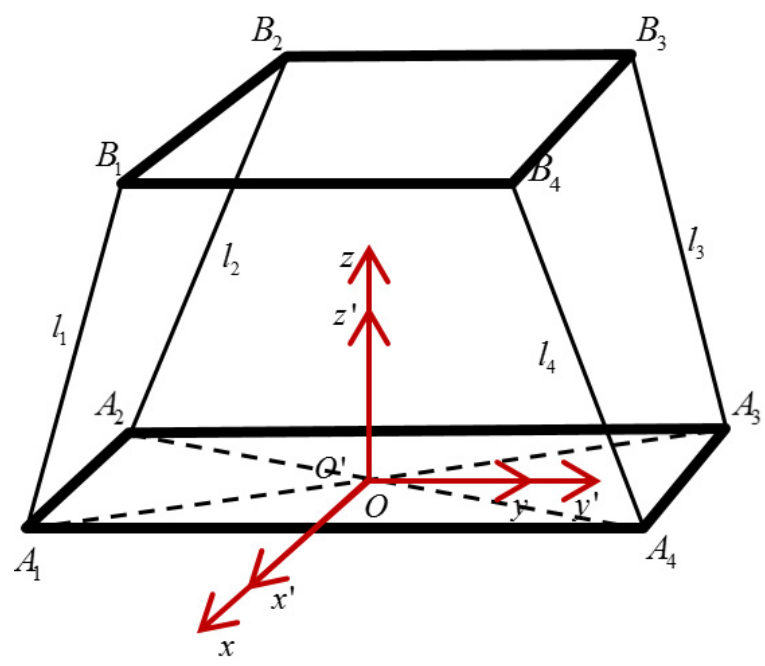

Figure 4. Simplified kinematic structure

Here, the rotation angles of the axes around $z, y, x$ are respectively $\alpha, \beta, \gamma$ according to z-y-x Euler Angle and the rotation matrix is

$$
\begin{aligned}
{ }_{O^{o}}^{o} R & =\operatorname{Rot}(z, \alpha) \operatorname{Rot}(y, \beta) \operatorname{Rot}(x, \gamma) \\
& =\left[\begin{array}{ccc}
c \alpha & -s \alpha & 0 \\
s \alpha & c \alpha & 0 \\
0 & 0 & 1
\end{array}\right]\left[\begin{array}{ccc}
c \beta & 0 & s \beta \\
0 & 1 & 0 \\
-s \beta & 0 & c \beta
\end{array}\right]\left[\begin{array}{ccc}
1 & 0 & 0 \\
0 & c \gamma & -s \gamma \\
0 & s \gamma & c \gamma
\end{array}\right] \\
& =\left[\begin{array}{ccc}
c \alpha c \beta & c \alpha s \beta s \gamma-s \alpha c \gamma & c \alpha s \beta c \gamma+s \alpha s \gamma \\
s \alpha c \beta & s \alpha s \beta s \gamma+c \alpha c \gamma & s \alpha s \beta c \gamma-c \alpha s \gamma \\
-s \beta & c \beta s \gamma & c \beta c \gamma
\end{array}\right]
\end{aligned}
$$

where $c \alpha$ denotes $\cos \alpha, s \alpha$ denotes $\sin \alpha$ and the rest are the same.

So, the coordinate transformation formula is the following

$$
B_{i}={ }_{O^{\prime}}^{o} R B_{i}{ }^{\prime}(i=1,2,3,4)
$$


The length of each limb $l_{i}$ can be obtained

$$
\begin{aligned}
l_{1}=\{ & {\left[\frac{1}{2} n_{B} c \alpha c \beta-\frac{1}{2} m_{B}(c \alpha s \beta s \gamma-s \alpha c \gamma)+h(\cos \beta c \gamma+s \alpha s \gamma)-\frac{1}{2} n_{A}\right]^{2} } \\
& +\left[\frac{1}{2} n_{B} s \alpha c \beta-\frac{1}{2} m_{B}(s \alpha s \beta s \gamma+c \alpha c \gamma)+h(\operatorname{sss} \beta c \gamma-c \alpha s \gamma)+\frac{1}{2} m_{A}\right]^{2} \\
& \left.+\left[-\frac{1}{2} n_{B} s \beta-\frac{1}{2} m_{B} c \beta s \gamma+h c \beta c \gamma\right]^{2}\right\}^{\frac{1}{2}}
\end{aligned}
$$$$
l_{2}=\left\{\left[-\frac{1}{2} n_{B} c \alpha c \beta-\frac{1}{2} m_{B}(c \alpha s \beta s \gamma-s \alpha c \gamma)+h(c \alpha s \beta c \gamma+s \alpha s \gamma)+\frac{1}{2} n_{A}\right]^{2}\right.
$$$$
+\left[-\frac{1}{2} n_{B} s \alpha c \beta-\frac{1}{2} m_{B}(s \alpha s \beta s \gamma+c \alpha c \gamma)+h(s \alpha s \beta c \gamma-c \alpha s \gamma)+\frac{1}{2} m_{A}\right]^{2}
$$$$
\left.+\left[\frac{1}{2} n_{B} s \beta-\frac{1}{2} m_{B} c \beta s \gamma+h c \beta c \gamma\right]^{2}\right\}^{\frac{1}{2}}
$$

$$
\begin{aligned}
l_{3}=\{ & {\left[-\frac{1}{2} n_{B} c \alpha c \beta+\frac{1}{2} m_{B}(c \alpha s \beta s \gamma-s \alpha c \gamma)+h(c \alpha s \beta c \gamma+s \alpha s \gamma)+\frac{1}{2} n_{A}\right]^{2} } \\
& +\left[-\frac{1}{2} n_{B} s \alpha c \beta+\frac{1}{2} m_{B}(s \alpha s \beta s \gamma+c \alpha c \gamma)+h(s \alpha s \beta c \gamma-c \alpha s \gamma)-\frac{1}{2} m_{A}\right]^{2} \\
& \left.+\left[\frac{1}{2} n_{B} s \beta+\frac{1}{2} m_{B} c \beta s \gamma+h c \beta c \gamma\right]^{2}\right\}^{\frac{1}{2}}
\end{aligned}
$$

$$
\begin{aligned}
l_{4}= & \left\{\left[\frac{1}{2} n_{B} c \alpha c \beta+\frac{1}{2} m_{B}(\cos \beta s \gamma-s \alpha c \gamma)+h(\cos \beta c \gamma+s \alpha s \gamma)-\frac{1}{2} n_{A}\right]^{2}\right. \\
& +\left[\frac{1}{2} n_{B} s \alpha c \beta+\frac{1}{2} m_{B}(s \alpha s \beta s \gamma+c \alpha c \gamma)+h(s \alpha s \beta c \gamma-c \alpha s \gamma)-\frac{1}{2} m_{A}\right]^{2} \\
& \left.+\left[-\frac{1}{2} n_{B} s \beta+\frac{1}{2} m_{B} c \beta s \gamma+h c \beta c \gamma\right]^{2}\right\}^{\frac{1}{2}}
\end{aligned}
$$

$$
\mathbf{J} \boldsymbol{\delta} \boldsymbol{\theta}_{\mathbf{k}}=-\mathbf{F}(\boldsymbol{\theta})
$$

Thus inverse kinematics of the mechanism is obtained. At present, the forward kinematics solution of parallel robot has not been fully solved yet (Dehghani et. al, 2008). A common method is to use numerical solutions based on algebraic equations. According to formula (9-12), a set of nonlinear equations can be obtained. In this paper, Newton-Raphson method (Ben-Israel, 1965) is chosen to solve the referred equations.

Firstly, the following function can be constructed as

$$
F_{i}(\theta)=\left\|\vec{l}_{i}\right\|-l_{i}(i=1 \ldots 4)
$$

According to Newton-Raphson method, the following iterative formula can be obtained:

$\theta_{k+1}=\theta_{k}+\delta \theta_{k}$ with $\left[\begin{array}{c}\mathbf{J}_{1} \\ \mathbf{J}_{2} \\ \mathbf{J}_{3} \\ \mathbf{J}_{4}\end{array}\right]_{4 \times 3}, \mathbf{F}(\boldsymbol{\theta})=\left[\begin{array}{l}\mathbf{F}_{1}(\boldsymbol{\theta}) \\ \mathbf{F}_{2}(\boldsymbol{\theta}) \\ \mathbf{F}_{3}(\boldsymbol{\theta}) \\ \mathbf{F}_{4}(\boldsymbol{\theta})\end{array}\right]_{4 \times 1}$

The following formula can be further obtained

$$
\delta \boldsymbol{\theta}_{\mathbf{k}}=-\mathbf{J}^{+} \mathbf{F}(\boldsymbol{\theta})
$$

where $\mathrm{J}^{+}$represents the pseudo-inverse of the matrix $\mathbf{J}$ :

$\mathbf{J}^{+}=\left(\mathbf{J}^{\mathrm{T}} \mathbf{J}\right)^{-1} \mathbf{J}^{\mathbf{T}}$

where $\boldsymbol{\theta}=[\alpha \beta \gamma]$ denotes the posture matrix of the moving platform, $\boldsymbol{\delta} \boldsymbol{\theta}_{\mathbf{k}}$ denotes the incremental value of the posture, $\mathbf{F}_{\mathbf{i}}(\boldsymbol{\theta})$ denotes the deviation function of the $i$ th pushrod, $\mathbf{J}_{\mathbf{i}}$ denotes the derivative of $\mathbf{F}_{\mathbf{i}}(\boldsymbol{\theta})$ with respect to $\theta$.

The initial guess is $\grave{\mathbf{e}}_{\mathbf{0}}=\left[\begin{array}{lll}\alpha_{0} & \beta_{0} & \gamma_{0}\end{array}\right]^{T}$ and $\boldsymbol{\delta} \boldsymbol{\theta}_{\mathbf{0}}$ can be obtained. Then use the formula $\boldsymbol{\theta}_{\mathbf{k}+1}=\boldsymbol{\theta}_{\mathbf{k}}+\boldsymbol{\delta} \boldsymbol{\theta}_{\mathbf{k}}$ repeatedly until $\left\|\boldsymbol{\delta}_{\boldsymbol{k}}\right\|<\xi$ where $\zeta$ represents the iterations of the error margin. The solution at this point is an approximation of the forward kinematics solution.

In order to verify the correctness of the forward kinematics and inverse kinematics, the length of the pushrods in four specific postures can be obtained by means of the MATLAB programming method, which is shown in Table 1.

With the result of inverse kinematics taken as the known condition, the posture of the moving platform can be obtained by using MATLAB , as it is shown in Table 2. 
Table 1: Inverse Kinematics

\begin{tabular}{|c|c|c|c|c|}
\hline $\begin{array}{c}\mathrm{rad} / \\
\mathrm{cm}\end{array}$ & 1 & 2 & 3 & 4 \\
\hline$\alpha$ & 0 & 0.1745 & 0.3491 & 0.5236 \\
\hline$\beta$ & 0 & 0.5236 & 1.0472 & 1.5708 \\
\hline$\gamma$ & 0 & 0.1745 & 0.3491 & 0.5236 \\
\hline$l_{1}$ & 27.1570 & 23.8500 & 22.2412 & 19.6850 \\
\hline$l_{2}$ & 27.1570 & 27.0247 & 28.3053 & 28.9396 \\
\hline$l_{3}$ & 27.1570 & 31.0518 & 31.1345 & 28.9396 \\
\hline$l_{4}$ & 27.1570 & 25.7325 & 21.9625 & 19.6850 \\
\hline
\end{tabular}

Table 2: Forward Kinematics

\begin{tabular}{|c|c|c|c|c|}
\hline $\begin{array}{c}\mathrm{cm} / \\
\mathrm{rad}\end{array}$ & 1 & 2 & 3 & 4 \\
\hline$l_{1}$ & 27.1570 & 23.8500 & 22.2412 & 19.6850 \\
\hline$l_{2}$ & 27.1570 & 27.0247 & 28.3053 & 28.9396 \\
\hline$l_{3}$ & 27.1570 & 31.0518 & 31.1345 & 28.9396 \\
\hline$l_{4}$ & 27.1570 & 25.7325 & 21.9625 & 19.6850 \\
\hline$\alpha$ & 0 & 0.1745 & 0.3491 & 0.5236 \\
\hline$\beta$ & 0 & 0.5236 & 1.0472 & 1.5708 \\
\hline$\gamma$ & 0 & 0.1745 & 0.3491 & 0.5236 \\
\hline
\end{tabular}

\subsection{Dynamic Model}

The main purpose of this section is to solve the dynamic inverse solution by using Newton-Euler formulation. Considering the waist a cylinder. The force analysis of the mechanism is shown in Figure 5. The dynamic equations of the system are listed as follows:

$$
\left\{\begin{array}{l}
\sum_{i=1}^{4} \mathbf{t}_{i}+\mathbf{f}_{N}+m \mathbf{g}+\mathbf{f}_{c}=0 \\
\sum_{i=1}^{4}\left({ }_{O^{o}} \mathbf{R} \overrightarrow{O^{\prime} B_{i}} \times \mathbf{t}_{i}\right)+\mathbf{M}_{r}+{ }_{O^{\prime}}^{o} \mathbf{R} \overrightarrow{O^{\prime} C} \times m \mathbf{g}+\mathbf{M}_{c}=0
\end{array}\right.
$$

where $\mathbf{t}_{i}(i=1,2,3,4)$ is the force exerted by the $i$ th pushrod to the human body, $\mathbf{f}_{N}$ is the support of the lower limbs to the trunk, $m$ is the quality of the waist moving platform, $\mathbf{f}_{c}$ is the inertial force, $\mathbf{M}_{r}$ is the rotational damping moment of waist, $\quad \mathbf{M}_{r}=\left[\begin{array}{lll}M_{r 1} & M_{r 2} & M_{r 3}\end{array}\right]^{T}$ with $M_{r i}=n_{i} \bullet \theta_{i}$, $n_{i}$ represents the damping coefficient of muscle for the waist rotation in three directions and $\mathbf{M}_{c}$ denotes the inertia moment.

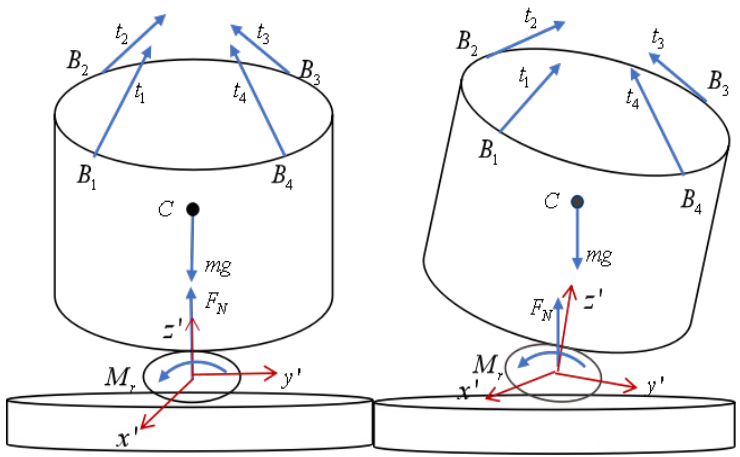

(a)

(b)

Figure 5. Force analysis of the mechanism((a) upright(b)bending)

According to Newton-Euler theorem,

$\mathbf{f}_{\mathrm{c}}=-m \mathbf{v}_{\mathrm{c}}$

$\mathbf{M}_{\mathrm{c}}=-\mathbf{I}_{\mathrm{c}} \omega-\omega \times\left(\mathbf{I}_{\mathrm{c}} \omega\right)$

where $\dot{\mathbf{v}}_{c}$ represents the acceleration of the centroid of the waist moving platform, $\mathbf{I}_{c}$ is moment of inertia of moving platform, $\omega$ denotes angular velocity of moving platform and $\omega$ denotes angular acceleration of moving platform.

Write equations (20) into matrix form:

$$
\mathbf{D T}=\mathbf{W}
$$

where

$\mathbf{D}=\left[\begin{array}{l}\mathbf{D}_{1} \\ \mathbf{D}_{2}\end{array}\right]=\left[\begin{array}{cccc}\mathbf{U}_{1} & \mathbf{U}_{2} & \mathbf{U}_{3} & \mathbf{U}_{4} \\ { }_{0}^{0} \mathbf{R} \overline{O^{\prime} B_{1}} \times \mathbf{U}_{1} & { }_{0}^{0} \bar{R} \overline{O^{\prime} B_{2}} \times \mathbf{U}_{2} & { }_{0}^{0} \bar{R} \overline{O^{\prime} B_{3}} \times \mathbf{U}_{3} & { }_{0}^{0} \overline{O^{\prime} B_{4}} \times \mathbf{U}_{4}\end{array}\right]$

$\mathbf{T}=\left[\begin{array}{llll}T_{1} & T_{2} & T_{3} & T_{4}\end{array}\right]^{T}$

$\mathbf{W}=\left[\begin{array}{c}\mathbf{W}_{1} \\ \mathbf{W}_{2}\end{array}\right]=\left[\begin{array}{c}\mathbf{f}_{N}+m \mathbf{g}+\mathbf{f}_{c} \\ \mathbf{M}_{r}+{ }_{o^{o}} \cdot \mathbf{R} \overrightarrow{O^{\prime} C} \times m \mathbf{g}+\mathbf{M}_{c}\end{array}\right]$

$\mathbf{U}_{i}=-\vec{L}_{i} / L_{i}$ represents a unit vector with the direction opposite to the direction of the pushrod force.

Since $\mathbf{f}_{N}$ is unmeasured, one can focus on $\mathbf{D}_{2} \mathbf{T}=\mathbf{W}_{2}$. Here the solution of the force can not be uniquely determined. Therefore, the distributed optimal solution of thrust applied to the waist must be solved. It is necessary to take the norm of $P>1$ as the optimization objective (Verhoeven \& Hiller, 2000). Here the optimization model is a nonlinear programming 
problem with the 2-norm of thrust applied to the waist as the optimization objective.

\section{Find $\mathbf{T}$}

$\operatorname{Min}\|\mathbf{T}\|_{2}$

s.t. $\mathbf{D}_{\mathbf{2}} \mathbf{T}=\mathbf{W}_{2}$

$\mathbf{T} \geq \mathbf{0}$

So far, the force exerted on the human body by the four pushrods during waist motion has been obtained as $\mathbf{T}=\left[\begin{array}{llll}T_{1} & T_{2} & T_{3} & T_{4}\end{array}\right]^{T}$, from which the value of the reacting force to the pushrods can be obtained as

$$
\mathbf{T}_{b}=\left[\begin{array}{llll}
T_{b 1} & T_{b 2} & T_{b 3} & T_{b 4}
\end{array}\right]^{T}=\left[\begin{array}{llll}
T_{1} & T_{2} & T_{3} & T_{4}
\end{array}\right]^{T}
$$

\subsection{Mathematical Model of Pushrod}

Since the length of the pushrod is proportional to the motor angular displacement, the angles of the four motors assumed to be $\theta_{i}(i=1,2,3,4)$, are taken as controlled variables. Considering that the four pushrods have the same models, the corresponding motor parameters are consistent. In each motor, the voltage balance equation can be written as

$U_{C i}(t)=L_{a} \frac{d I_{a i}(t)}{d t}+R_{a} I_{a i}(t)+U_{a i}(t)$

$U_{a i}(t)=C_{e} w_{i}(t)$

The moment balance equation can be written as

$T_{m i}(t)=J \frac{d w_{i}(t)}{d t}+F_{f i}(t)+T_{b i}(t)+D_{i}$

$T_{m i}(t)=K_{m} I_{a i}(t)$

where $U_{C i}(i=1, \ldots, 4)$ is the armature voltage of the four motors, $L_{a}$ is the winding inductance which is small enough to be ignored, $I_{a i}(i=1, \ldots, 4)$ denotes the armature current of each motor, $R_{a}$ is the winding resistance which is uniform in the four motors, $U_{a i}(i=1, \ldots, 4)$ is the respective armature back electromotive force, $K_{e}$ is the back electromotive force constant, $w_{i}=\dot{\theta}_{i}(i=1, \ldots, 4)$ denotes the motor angular velocity, $T_{m i}(i=1, \ldots, 4)$ is the motor torque, $J$ denotes the total moment of inertia, $T_{b i}(i=1, \ldots, 4)$ denotes the load moment applied by human body to the pushrods, $D_{i}$ is the external disturbance, $K_{m}$ denotes the constant moment and $F_{f i}(i=1, \ldots, 4)$ denotes the friction moment applied to each motor, which in this paper is described in LuGre model due to its accuracy in both dynamic and static characteristics. Considering the parameter uncertainties, a modified LuGre model is proposed in the next section.

The dynamic of pushrod can be modified as

$J \ddot{\theta}_{i}=\frac{K_{m}}{R_{a}} U_{C i}-\frac{K_{e} \cdot K_{m}}{R_{a}} \dot{\theta}_{i}-F_{f i}-T_{b i}-D_{i}$

Let $x_{i}=\dot{\theta}_{i}, u_{i}=U_{C i}(i=1, \ldots 4)$, it can be written into $\dot{x}_{i}=a x_{i}+b u_{i}+\Delta_{i}$

with $a=-\frac{K_{e} \cdot K_{m}}{J R_{a}}, \quad b=\frac{K_{m}}{J R_{a}}, \Delta_{i}=-\frac{F_{f i}+T_{b i}+D_{i}}{J}$.

\subsection{Modified LuGre model}

To describe different experimentally observed friction phenomena, several friction models with a varying degree of complexity have been proposed in the specialized literature over the past few decades. These friction models can be divided into three categories according to their functional forms: (i)static friction models, (ii) dynamic friction models and (iii) accelerationdependent friction models. Static friction models depend only on the relative velocity of the surfaces in frictional contact, and include the Stribeck friction model and the Coulomb friction model. Since static friction models cannot capture the hysteresis in the friction force, dynamic friction models which depend on some internal variables and the acceleration-dependent friction models with the force depending on the relative acceleration are developed.

In this work, the LuGre model is selected to describe the friction in the system because it can perfectly explain both the dynamic and static characteristics of the friction. The original LuGre model was proposed by Canudas de Wit (Canudas et al., 1995). The model relies on estimating the average deflection $z$ of the microscopic asperities (bristles) between the two contacting surfaces, which is achieved by means of the following nonlinear state observer.

$\dot{z}=w-\frac{|w|}{g(w)} z$ 
where $g(v)$ is a positive function defined as

$$
g(w)=\frac{1}{\sigma_{0}}\left[F_{c}+\left(F_{s}-F_{c}\right) e^{-\left(\frac{w}{w_{s}}\right)^{2}}\right]
$$

where $F_{c}$ is the Coulomb friction force, $F_{s}$ is the static friction force, and $w_{s}$ is the Stribeck velocity.

Based on the state $\mathrm{z}$, the friction $f$ acting between two surfaces with relative velocity $w$ can be represented as

$$
F=\sigma_{0} z+\sigma_{1} \dot{z}+\sigma_{2} w
$$

where $\sigma_{0}$ is the stiffness coefficient, $\sigma_{1}$ is the damping coefficient, and $\sigma_{2}$ is viscous friction coefficient.

Considering that the friction parameter will vary with the external environment, the parameters $\mu$ and $v$ are introduced to correct the LuGre model. The modified LuGre model is defined as

$$
\begin{aligned}
F_{f} & =\mu\left(\sigma_{0} z+\sigma_{1} \dot{z}\right)+v \sigma_{2} w \\
& =\mu\left(\sigma_{0} z+\sigma_{1}\left(w-\frac{|w|}{g(w)} z\right)\right)+v \sigma_{2} w \\
& =\mu z\left(\sigma_{0}-\sigma_{1} \frac{|w|}{g(w)}\right)+\left(\mu \sigma_{1}+v \sigma_{2}\right) w
\end{aligned}
$$

where $\mu$ is used to reflect changes in the average deflection of bristles, $v$ is used to reflect changes in the coefficient of viscous friction. They meet

$$
0<\mu_{\min }<\mu<\mu_{\max }, 0<v_{\min }<v<v_{\max }
$$

\section{Robust Adaptive Integral Backstepping Cooperative Control of Lower Back Exoskeleton}

Since the lower back exoskeleton is equipped with four motors, the synchronous control is important for interrelated multi-motors to ensure their coordination work. On the other hand, it is of equal importance to realize high precision tracking of each pushrod. In this paper, a robust adaptive integral backstepping cooperative controller is proposed to control the lower back exoskeleton system. The structure of the controller is shown in Figure 6.

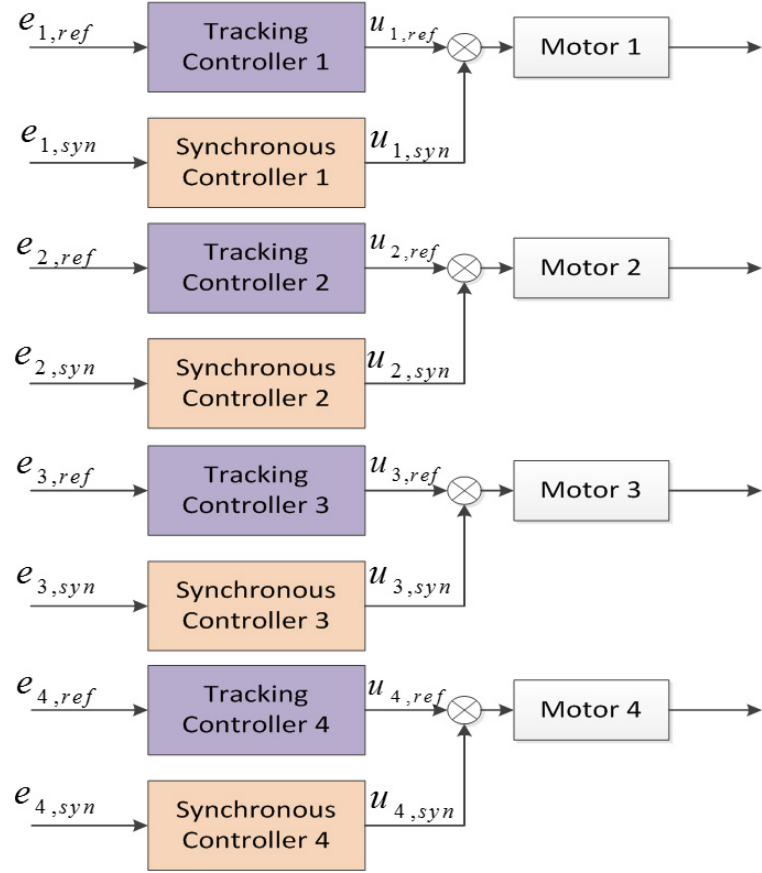

Figure 6. The control structure of the whole system

The structure of tracking controller of motor $i$ is shown in Figure 7 and includes two parts. $C_{i, \text { erf }}$ is used to track the desired displacement signal accurately and $C_{i, \text { mean }}$ is used to compensate the mean displacement error of the controlled motor $i$.

It can be seen that the complete control effort of motor $i$ is

$u_{i}=u_{i, r e f}+u_{i, s y n}$

where $u_{i, \text { ref }}$ is the control effort for angle tracking of motor $i, u_{i, \text { mean }}$ is the synchronous control part of motor $i$.

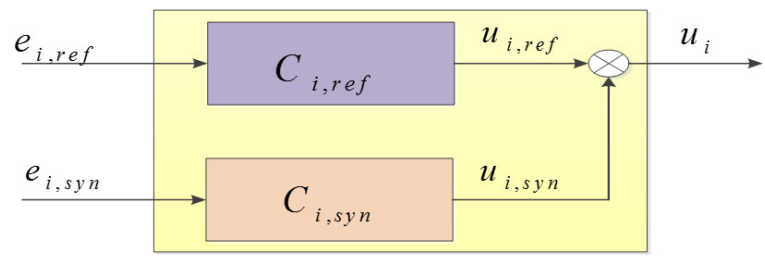

Figure 7. The structure of the complete controller of motor $i$

\subsection{Mean Deviation Coupling Control}

This section focuses on the synchronous controller design in order to reduce the synchronization error of output displacement of the motors. Since the traditional mean deviation coupling control ( $\mathrm{Li}$, Sun and Zhang, 2016) is based on the same given references, an improved mean deviation coupling control strategy is proposed in this paper. 
The schematic diagram of mean deviation coupling control strategy is shown in Figure 8. The angle tracking error of motor $i$ is defined as

$$
e_{i, r e f}(t)=\theta_{i}^{*}(t)-\theta_{i}(t) \quad(i=1,2, \ldots 4)
$$

where $\theta_{i}^{*}(t)(i=1, \ldots 4)$ is the desired reference angle, $\theta_{i}(t)(i=1, \ldots 4)$ is the actual angle of each motor.

Take the reference angle of motor 1 as the base, the ratio of the other motors to motor 1 can be calculated as

$r_{i 1}=\frac{\theta_{i}^{*}}{\theta_{1}^{*}} \quad(i=1,2, \ldots 4)$

The angle synchronization error of motor $i$ with motor $j$ is defined as

$e_{i, j}(t)=\frac{\theta_{i}(t)}{r_{i 1}}-\frac{\theta_{j}(t)}{r_{j 1}} \quad(i, j=1,2, \ldots 4)$

Define the mean synchronization angle error of motor $i$ with other motors in the following way

$e_{i, s y n}(t)=\theta_{i}(t)-\theta_{\text {mean }}(t)=\theta_{i}(t)-\frac{\sum_{j=1}^{4} \frac{\theta_{j}(t)}{r_{j 1}}}{4}(i, j=1,2, \ldots 4)$

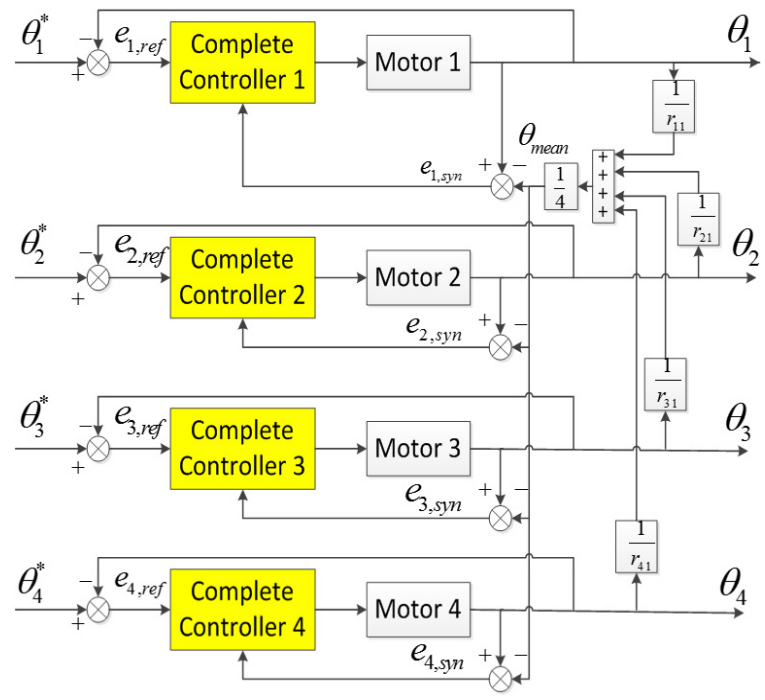

Figure 8. The schematic diagram of mean deviation coupling control

The derivative of the mean synchronization angle error $e_{i, s y n}(t)$ is

$\dot{e}_{i, s y n}(t)=\dot{\theta}_{i}(t)-\frac{\sum_{j=1}^{4} \frac{\dot{\theta}_{j}(t)}{r_{j 1}}}{4} \quad(i=1, \ldots, 4)$
The second order derivative of the mean synchronization angle error $e_{i, s y n}(t)$ is

$$
\begin{aligned}
\ddot{e}_{i, s y n}(t) & =\dot{x}_{i}(t)-\frac{\sum_{j=1}^{4} \frac{\dot{x}_{j}(t)}{r_{j 1}}}{4}=a_{i} x_{i}(t)+b_{i} u_{i}(t)+\Delta_{i}-\frac{\sum_{j=1}^{4} \frac{a_{j} x_{j}(t)+b_{j} u_{j}(t)+\Delta_{j}}{r_{j 1}}}{4} \\
& =a x_{i}(t)+b u_{i}(t)+\Delta_{i}-\frac{a}{4} \sum_{j=1}^{4} \frac{x_{j}(t)}{r_{j 1}}-\frac{b}{4} \sum_{j=1}^{4} \frac{u_{j}(t)}{r_{j 1}}-\frac{1}{4} \sum_{j=1}^{4} \frac{\Delta_{j}}{r_{j 1}} \\
& =a\left(x_{i}(t)-\frac{1}{4} \sum_{j=1}^{4} \frac{x_{j}(t)}{r_{j 1}}\right)+b\left(u_{i}(t)-\frac{1}{4} \sum_{j=1}^{4} \frac{u_{j}(t)}{r_{j 1}}\right)+\Delta_{i}-\frac{1}{4} \sum_{j=1}^{4} \frac{\Delta_{j}}{r_{j 1}} \\
& =a \dot{e}_{i, \text { mean }}(t)+b u_{i, \text { mean }}(t)+\Delta_{i}-\frac{1}{4} \sum_{j=1}^{4} \frac{\Delta_{j}}{r_{j 1}}
\end{aligned}
$$

with $a=a_{i}=a_{j}, b=b_{i}=b_{j}(i, j=1, \ldots, 4)$.

A generalized sliding surface is defined as follows

$s_{i, s y n}(t)=c e_{i, s y n}(t)+\dot{e}_{i, s y n}(t)$

where $c$ is a given positive constant.

Then the following equation can be obtained

$\dot{s}_{i, s y n}(t)=c \dot{e}_{i, s y n}(t)+\ddot{e}_{i, s y n}(t)$

$$
=(c+a) \dot{e}_{i, s y n}(t)+b u_{i, s y n}(t)+\Delta_{i}-\frac{1}{4} \sum_{j=1}^{4} \frac{\Delta_{j}}{r_{j 1}}
$$

The mean synchronization deviation SMC law is $u_{i, s y n}(t)=u_{i s y n, e q}(t)+u_{i s y n, \text { rea }}(t)$

The equivalent control

$u_{i s y n, e q}(t)=-\frac{1}{b}\left(c \dot{e}_{i, s y n}(t)+a \dot{e}_{i, s y n}(t)\right)$

The reaching control part of control effort

$u_{\text {iref, rea }}(t)=\frac{1}{b}\left(k s_{i, s y n}(t)+\rho_{i, s y n} \operatorname{sgn}\left(s_{i, s y n}(t)\right)\right)$

where $k$ is a positive constant, $\rho_{i, \text { ref }}$ is the switched gain.

\subsection{Angle Tracking Controller based on Nonlinear Observer Design}

In this paper, the robust adaptive integral backstepping control is chosen to realize the angle tracking. The following equation can be obtained by taking $F_{f}$ into equation (32)

$J \ddot{\theta}_{i}=\frac{K_{m}}{R_{a}} u_{i}-\frac{K_{e} \cdot K_{m}}{R_{a}} \dot{\theta}_{i}-\mu_{i}\left(\sigma_{0} z_{i}+\sigma_{1} \dot{z}_{i}\right)-v_{i} \sigma_{2} w_{i}-T_{b i}-D_{i}$

The angle tracking error of each motor is defined as $e_{i, \text { ref. }}=\theta_{i .}^{*}-\theta_{i}(i=1, \ldots 4)$ and its derivative is $\dot{e}_{i, r e f}=\dot{\theta}_{i}^{*}-\dot{\theta}_{i}(i=1, \ldots, 4)$. The Lyapunov function can be defined as follows 
$V_{1 i}\left(e_{i, r e f}\right)=\frac{1}{2} e_{i, r e f}^{2}(i=1, \ldots 4)$

The derivative of Lyapunov function is computed as below.

$\dot{V}_{1 i}\left(e_{i, r e f}\right)=e_{i, r e f} \dot{e}_{i, r e f}=e_{i, r e f}\left(\dot{\theta}_{i}^{*}-\dot{\theta}_{i}\right)$

The virtual control of the $i$ th motor $\dot{\rho}_{d i}(i=1, \ldots 4)$ is chosen as follows

$\dot{\rho}_{d i}=\dot{\theta}_{i}^{*}+h_{1 i} e_{i, r e f}+h_{2 i} \varphi_{i}\left(h_{1 i}>0, h_{2 i}>0\right)$

where $h_{1 i}, h_{2 i}(i=1, \ldots 4)_{t}$ are positive constants of the $i$ th motor and $\varphi_{i}=\int_{0}^{1} e_{i, r e f} d t(i=1, \ldots 4)$

Then the second error of the $i$ th motor is defined as

$$
\begin{aligned}
e_{i, \text { ref } 2} & =\dot{\rho}_{d i}-\dot{\theta}_{i}=\dot{\theta}_{i}^{*}+h_{1 i} e_{i, \text { ref }}+h_{2 i} \varphi_{i}-\dot{\theta}_{i} \\
& =\dot{e}_{i, \text { ref }}+h_{1 i} e_{i, r e f}+h_{2 i} \varphi_{i}
\end{aligned}
$$

$\dot{e}_{i, r e f}$ can be rewritten as follows

$\dot{e}_{i, \text { ref }}=e_{i, \text { ref } 2}-h_{1 i} e_{i, \text { ref }}-h_{2 i} \varphi_{i}$

The derivative of $e_{i, \text { ref } 2}$ is

$$
\dot{e}_{i, r e f 2}=\ddot{\theta}_{i}^{*}-\ddot{\theta}_{i}+h_{1 i} \dot{e}_{i, \text { ref }}+h_{2 i} e_{i, r e f}
$$

Then the following equation can be obtained

$$
\begin{aligned}
& J \dot{e}_{i, r e f 2}=J \ddot{\theta}_{i}^{*}-J \ddot{\theta}_{i}+J h_{1 i} \dot{e}_{i, \text { ref }}+J h_{2 i} e_{i, r e f} \\
&=J \ddot{\theta}_{i}^{*}-\frac{K_{m}}{R_{a}} u_{i}+\frac{K_{e} \cdot K_{m}}{R_{a}} w_{i}+\mu_{i}\left(\sigma_{0} z_{i}+\sigma_{1} \dot{z}_{i}\right)+v_{i} \sigma_{2} w_{i} \\
&+T_{b i}+D_{i}+J h_{1 i} \dot{e}_{i, r e f}+J h_{2 i} e_{i, r e f} \\
&= J \ddot{\theta}_{i}^{*}-\frac{K_{m}}{R_{a}} u_{i}+\frac{K_{e} \cdot K_{m}}{R_{a}} w_{i}+\mu_{i} z_{i}\left(\sigma_{0}-\sigma_{1} \frac{\left|w_{i}\right|}{g\left(w_{i}\right)}\right)+\left(\mu_{i} \sigma_{1}+v_{i} \sigma_{2}\right) w_{i} \\
&+T_{b i}+D_{i}+J h_{1 i} \dot{e}_{i, r e f}+J h_{2 i} e_{i, \text { ref }}
\end{aligned}
$$

Since the parameters $\mu_{i}, v_{i}$ and $D_{i}(i=1, \ldots 4)$ of the system are uncertain, they need to be estimated by the adaptive algorithm. $\hat{\mu}_{i}, \hat{v}_{i}$ and $\hat{D}_{i}(i=1, \ldots, 4)$ are the estimated values of $\mu_{i}, v_{i}$ and $D_{i}(i=1, \ldots, 4)$ respectively. The error can be defined as follows

$$
\tilde{D}_{i}=D_{i}-\hat{D}_{i}, \quad \tilde{\mu}_{i}=\mu_{i}-\hat{\mu}_{i}, \quad \tilde{v}_{i}=v_{i}-\hat{v}_{i}
$$

In addition, due to the unmeasured state $z_{i}(i=1, \ldots 4)$ of the friction model in the four motors, a nonlinear observer for the $i$ th motor is defined as

$$
\dot{\hat{z}}_{i}=w_{i}-\frac{\left|w_{i}\right|}{g\left(w_{i}\right)} z_{i}+h_{3_{i}} e_{i, \text { ref } 2}\left(\sigma_{0}-\sigma_{1} \frac{\left|w_{i}\right|}{g\left(w_{i}\right)}\right)
$$

where $\hat{z}_{i}$ is the estimated value of $z_{i}, h_{3 i}(i=1, \ldots, 4)$ is a positive constant. The error of state observer is $\tilde{z}_{i}=z_{i}-\hat{z}_{i}$

The second Lyapunov function of the $i$ th motor is defined as

$$
\begin{gathered}
V_{2 i}\left(e_{i, r e f}, e_{i, r e f 2}\right)=V_{1 i}+\frac{1}{2} h_{2 i} \varphi_{i}^{2}+\frac{1}{2} h_{3 i} J e_{i, r e f 2}^{2}+\frac{1}{2} \mu_{i} \tilde{z}_{i}^{2}+\frac{1}{2 \eta_{1 i}} \tilde{D}_{i}^{2} \\
+\frac{1}{2 \eta_{2 i}} \tilde{\mu}_{i}^{2}+\frac{1}{2 \eta_{3 i}} \tilde{v}_{i}^{2}
\end{gathered}
$$

The derivative of $V_{2 i}$ is

$$
\begin{aligned}
& \dot{V}_{2 i}\left(e_{i, r e f}, e_{i, r e f}\right)=e_{i, r e f} \dot{e}_{i, r e f}+h_{2 i} \varphi_{i} e_{i, r e f}+h_{3 i} J e_{i, r e f} \dot{e}_{2 i} \\
& +\mu_{i} \tilde{z}_{i} \dot{\tilde{z}}_{i}+\frac{1}{\eta_{1 i}} \tilde{D}_{i} \dot{\tilde{D}}_{i}+\frac{1}{\eta_{2 i}} \tilde{u}_{i} \dot{\bar{\mu}}_{i}+\frac{1}{\eta_{3 i}} \tilde{r}_{i} \dot{\bar{v}}_{i} \\
& =e_{i, \text { ref }}\left(e_{i, r e f 2}-h_{1 i} e_{i, \text { ref }}-h_{2 i} \varphi_{i}\right)+h_{2 i} \varphi_{i} e_{i, \text { ref }} \\
& +e_{i, r e f 2} h_{3 i}\left(J \ddot{\theta}_{i}^{*}-\frac{K_{m}}{R_{a}} u_{i}+\frac{K_{e} \cdot K_{m}}{R_{a}} w_{i}\right. \\
& +\mu_{i} z_{i}\left(\sigma_{0}-\sigma_{1} \frac{\left|w_{i}\right|}{g\left(w_{i}\right)}\right)+\left(\mu_{i} \sigma_{1}+v_{i} \sigma_{2}\right) w_{i} \\
& \left.+T_{b i}+D_{i}+J h_{i} \dot{e}_{i, r e f}+J h_{2 i} e_{i, r e f}\right)+\mu_{i} \tilde{z}_{i} \dot{\tilde{z}}_{i} \\
& +\frac{1}{\eta_{1 i}} \tilde{D}_{i} \dot{\tilde{D}}_{i}+\frac{1}{\eta_{2 i}} \tilde{\mu}_{i} \dot{\tilde{\mu}}_{i}+\frac{1}{\eta_{3 i}} \tilde{r}_{i} \dot{\vec{v}}_{i} \\
& =-h_{i i} e_{i, r e f}^{2}+e_{i, r f} e_{i, r e f 2}+e_{i, r e f 2} h_{3 i}\left(J \ddot{\theta}_{i}^{*}-\frac{K_{m}}{R_{a}} u_{i}\right. \\
& +\frac{K_{e} \cdot K_{m}}{R_{a}} w_{i}+\mu_{i} z_{i}\left(\sigma_{0}-\sigma_{1} \frac{\left|w_{i}\right|}{g\left(w_{i}\right)}\right) \\
& \left.+\left(\mu_{i} \sigma_{1}+v_{i} \sigma_{2}\right) w_{i}+T_{b i}+D_{i}+J h_{1 i} \dot{e}_{i, r e f}+J h_{2 i} e_{i, r e f}\right) \\
& +\mu_{i} \tilde{z}_{i}\left(-\frac{\left|w_{i}\right|}{g\left(w_{i}\right)} \tilde{z}_{i}-h_{3 i} e_{i, \text { ref } 2}\left(\sigma_{0}-\sigma_{1} \frac{\left|w_{i}\right|}{g\left(w_{i}\right)}\right)\right) \\
& +\frac{1}{\eta_{1 i}} \tilde{D}_{i} \dot{\tilde{D}}_{i}+\frac{1}{\eta_{2 i}} \tilde{\mu}_{i} \dot{\tilde{\mu}}_{i}+\frac{1}{\eta_{3 i}} \tilde{v}_{i} \dot{\bar{v}}_{i}
\end{aligned}
$$

The actual control input of the $i$ th motor is achieved as follows

$$
\begin{aligned}
u_{i}= & \frac{R_{a}}{K_{m}}\left(\frac{K_{m} K_{e}}{R_{a}} w_{i}+J \ddot{\theta}_{i}^{*}+J h_{1 i} \dot{e}_{i, r e f}+J h_{2 i} e_{i, r e f}+\frac{e_{i, \text { ref }}}{h_{3 i}}+h_{4 i} e_{i, r e f 2}\right. \\
& \left.+\hat{\mu}_{i} \hat{v}_{i}\left(\sigma_{0}-\sigma_{1} \frac{\left|w_{i}\right|}{g\left(w_{i}\right)}\right)+\left(\mu_{i} \sigma_{1}+v_{i} \sigma_{2}\right) w_{i}+\hat{D}_{i}+T_{b i}\right)
\end{aligned}
$$

By combining (55) and (56) the following formula can be obtained

$$
\begin{array}{r}
\dot{V}_{2 i}\left(e_{i, \text { ref }}, e_{i, \text { ref } 2}\right)=-h_{1 i} e_{i, \text { ref }}^{2}-h_{3 i} h_{4 i} e_{i, \text { ref } 2}^{2}-\mu_{i} \frac{\left|w_{i}\right|}{g\left(w_{i}\right)} \tilde{z}_{i}^{2}+h_{3 i} e_{i, \text { ref } 2} \tilde{D}_{i} \\
+h_{3 i} e_{i, \text { ref } 2}\left(\left(\mu_{i} z_{i}-\hat{\mu}_{i} \hat{z}_{i}\right)\left(\sigma_{0}-\sigma_{1} \frac{\left|w_{i}\right|}{g\left(w_{i}\right)}\right)+\left(\tilde{\mu}_{i} \sigma_{1}+\tilde{v}_{i} \sigma_{2}\right) w_{i}\right) \\
-h_{3 i} e_{i, r e f} \mu_{i} \tilde{z}_{i}\left(\sigma_{0}-\sigma_{1} \frac{\left|w_{i}\right|}{g\left(w_{i}\right)}\right)-\frac{1}{\eta_{1 i}} \tilde{D}_{i} \dot{\hat{D}}_{i}-\frac{1}{\eta_{2 i}} \tilde{\mu}_{i} \dot{\hat{\mu}}_{i}-\frac{1}{\eta_{3 i}} \tilde{v}_{i} \dot{\hat{v}}_{i}
\end{array}
$$


where $\mu_{i} z_{i}-\hat{\mu}_{i} \hat{z}_{i}=\mu_{i} \tilde{z}_{i}+\tilde{\mu}_{i} z_{i}$.

Then (57) can be achieved as follows

$$
\begin{aligned}
\dot{V}_{2 i}\left(e_{i, \text { ref }}, e_{i, \text { ref } 2}\right)=-h_{1 i} e_{i, \text { ref }}^{2}-h_{3 i} h_{4 i} e_{i, \text { ref } 2}^{2}-\mu_{i} \frac{\left|w_{i}\right|}{g\left(w_{i}\right)} \tilde{z}_{i}^{2}+h_{3 i} e_{i, \text { ref } 2} \tilde{D}_{i} \\
\quad+h_{3 i} e_{i, \text { ref } 2}\left(\left(\mu_{i} \tilde{z}_{i}+\tilde{\mu}_{i} \hat{z}_{i}\right)\left(\sigma_{0}-\sigma_{1} \frac{\left|w_{i}\right|}{g\left(w_{i}\right)}\right)+\left(\tilde{\mu}_{i} \sigma_{1}+\tilde{v}_{i} \sigma_{2}\right) w_{i}\right) \\
\quad-h_{3 i} e_{i, r e f 2} \mu_{i} \tilde{z}_{i}\left(\sigma_{0}-\sigma_{1} \frac{\left|w_{i}\right|}{g\left(w_{i}\right)}\right)-\frac{1}{\eta_{1 i}} \tilde{D}_{i} \dot{\hat{D}}_{i}-\frac{1}{\eta_{2 i}} \tilde{\mu}_{i} \dot{\hat{\mu}}_{i}-\frac{1}{\eta_{3 i}} \tilde{v}_{i} \dot{\hat{v}}_{i} \\
=-h_{1 i} e_{i, \text { ref }}^{2}-h_{3 i} h_{4 i} e_{i, \text { ref } 2}^{2}-\mu_{i} \frac{\left|w_{i}\right|}{g\left(w_{i}\right)} \tilde{z}_{i}^{2}+\tilde{D}_{i}\left(h_{3 i} e_{i, r e f 2}-\frac{1}{\eta_{1 i}} \dot{\hat{D}}_{i}\right) \\
+\tilde{\mu}_{i}\left(h_{3 i} e_{i, \text { ref } 2}\left(\left(\sigma_{0}-\sigma_{1} \frac{\left|w_{i}\right|}{g\left(w_{i}\right)}\right) \hat{z}_{i}+\sigma_{1} w_{i}\right)-\frac{1}{\eta_{2 i}} \dot{\hat{\mu}}_{i}\right) \\
+\tilde{v}_{i}\left(h_{3 i} e_{i, \text { ref } 2} \sigma_{2} w_{i}-\frac{1}{\eta_{3 i}} \dot{\hat{v}}_{i}\right)
\end{aligned}
$$

The adaptive rate is designed as below

$$
\left\{\begin{array}{l}
\dot{\hat{D}}_{i}=\eta_{1 i} h_{3 i} e_{i, \text { ref } 2} \\
\dot{\hat{\mu}}_{i}=\eta_{2 i} h_{3 i} e_{i, \text { ref } 2}\left(\hat{z}_{i}\left(\sigma_{0}-\sigma_{1} \frac{\left|w_{i}\right|}{g\left(w_{i}\right)}\right)+\sigma_{1} w_{i}\right) \\
\dot{\hat{v}}_{i}=\eta_{3 i} h_{3 i} e_{i, \text { ref } 2} \sigma_{2} w_{i}
\end{array}\right.
$$

Then

$\dot{V}_{2 i}\left(e_{i, \text { ref }}, e_{i, r \text { ref } 2}\right)=-h_{1 i} e_{i, \text { ref }}^{2}-h_{3 i} h_{4 i} e_{i, r e f 2}^{2}-\mu_{i} \frac{\left|w_{i}\right|}{g\left(w_{i}\right)} \tilde{z}_{i}^{2}$

where $g\left(w_{i}\right)>0$ and $\left|w_{i}\right|>0$, thus

$\dot{V}_{2 i}\left(e_{i, r e f}, e_{i, r e f 2}\right)<0$

From equations (44) and (54), it is clear that $V_{2 i}\left(e_{i, \text { ref }}, e_{i, \text { ref } 2}\right)$ is positive definite and $\dot{V}_{2 i}\left(e_{i, \text { ref }}, e_{i, \text { ref } 2}\right)$ is negative semi-definite. It can be concluded that $V_{2 i}\left(e_{i, r e f}, e_{i, r e f 2}\right)$ is bounded, which means that the variables $e_{i, r e f}, e_{i, \text { ref } 2}, \varphi_{i}, \tilde{z}_{i}, \tilde{D}_{i}, \tilde{\mu}_{i}, \tilde{v}_{i}$ are all bounded. On the basis of this analysis, it is obvious that the angle $\theta_{i}$, the angle speed $\dot{\theta}_{i}$ and the control input $u_{i}$ are all globally and uniformly bounded. It can also be verified that the closedloop system is asymptotically stable and the angle error approaches to zero also asymptotically by using the Lyapunov function.

\section{Validation of Effectiveness in the Power Assist Suit}

In this section, the issue of whether the proposed lower back exoskeleton can really assist human motion and reduce burden of the waist is discussed. At present, more and more scholars pay attention to the method of establishing the humanmachine coupling system model by using the human biomechanics software. This could be used to stimulate the dynamic behavior of human body movement in order to explore the biomechanics law in the process of human body movement.

Anybody is a human body modeling and simulation software which focus on biomechanical analysis. The Anybody human biomechanics software establishes the model on the base of the AnyScript programming language (Damsgaard et al., 2006). It can simultaneously complete the reverse dynamics analysis of the human skeleton and muscles, with results showing the force of the segments, the muscles and the joints as well as the activity of the muscles in the human body model.

In order to verify the effectiveness of the proposed lower back exoskeleton, a standing human model in initial posture is loaded and the waist motion of flexion and extension are defined during which the software can calculate the force in lumbar muscles. Anybody allows load to be added to a particular point on the model, which makes it feasible to compare the activity and the force of lumbar muscles with power assisted motion and with independent motion respectively.

The waist motion is coordinated by muscle groups including rectus abdominis, internal oblique, external oblique, erector spinae, quadratus lumborum, etc. In this paper, erector spinae is paid great attention due to its significance in supporting waist motion especially in flexion and extension.

\section{Simulation and Results}

\subsection{Results of Kinematics}

In this paper, the following three kinds of lumbar rehabilitation movements are put forward. The equation of lateral flexion is as follows

$\gamma=A_{x} \sin \left(\frac{2 \pi}{T_{x}} t+\varphi_{x}\right)$

The equation of flexion and extension is as follows:

$$
\beta= \begin{cases}A_{y 1} \sin \left(\frac{2 \pi}{T_{y}} t+\varphi_{y}\right) & n T_{y} \leq t \leq \frac{T_{y}}{2}+n T_{y} \\ A_{y 2} \sin \left(\frac{2 \pi}{T_{y}} t+\varphi_{y}\right) & \frac{T_{y}}{2}+n T_{y} \leq t \leq T_{y}+n T_{y}\end{cases}
$$


The equation of rotation is as follows

$\alpha=A_{z} \sin \left(\frac{2 \pi}{T_{z}} t+\varphi_{z}\right)$

where $A_{y_{1}}, A_{y_{2}}, A_{x}, A_{z}$ denote the amplitude of flexion, extension, lateral bending and rotation, $\varphi_{y}, \varphi_{x}, \varphi_{z}$ denote the initial phases (zero phases when standing normal) and $T_{y}, T_{x}, T_{z}$ denote the periods of time.

In the following simulation, the moving platform moves according with the motion shown in formula (50)

$\left\{\begin{array}{l}\alpha=\frac{\pi}{6} \sin \left(\frac{\pi}{10} t\right) \\ \beta= \begin{cases}\frac{5 \pi}{9} \sin \left(\frac{\pi}{10} t\right) & 20 n \leq t \leq 10+20 n \\ \frac{\pi}{6} \sin \left(\frac{\pi}{10} t\right) & 10+20 n \leq t \leq 20+20 n\end{cases} \\ \gamma=\frac{2 \pi}{9} \sin \left(\frac{\pi}{10} t\right)\end{array}\right.$

This device always generates aiding force to human body and the degree of waist motion is automatically adjusted by the electric motor control. Figure 9 shows the variation trend in the length of the four actuators, when the moving platform moves according with formula (69).

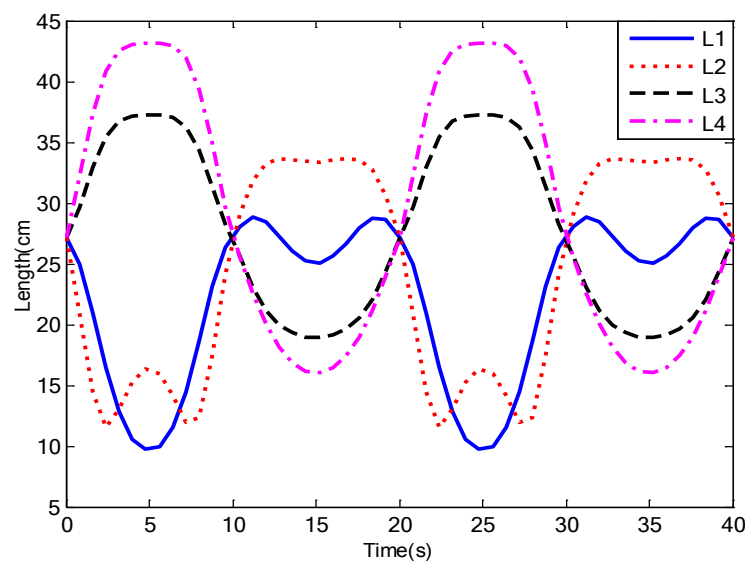

Figure 9. The variation trend in the length of the four actuators

\subsection{Results of Dynamics}

The variation trend in the force of the four pushrods exerted to human during motion (50) can be obtained as Figure 10 illustrates.

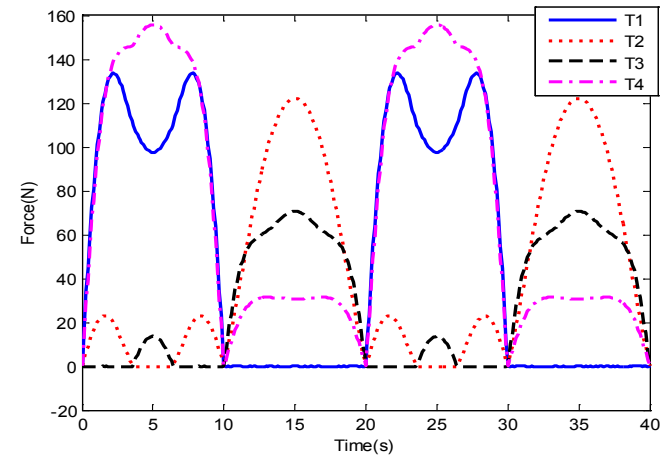

Figure 10. The force of the pushrod in motion

\subsection{Results of Robust Adaptive Integral Backstepping Control}

The displacement tracking error of the four motors in the control considering the changing friction is shown in the Figure 11.

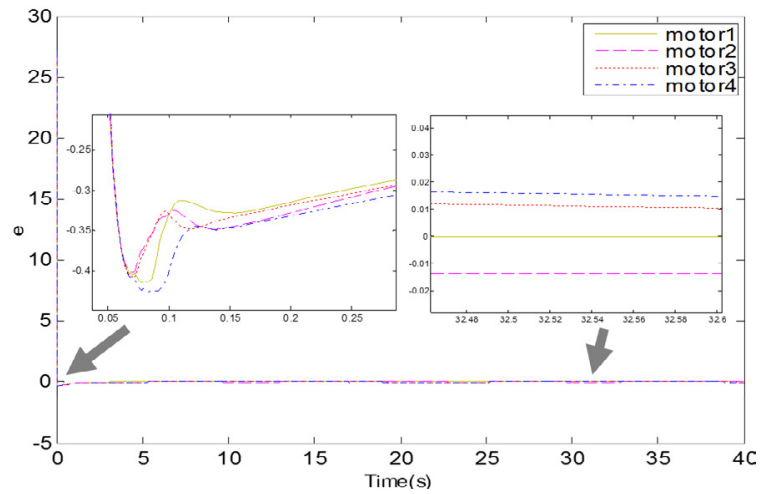

Figure 11. The angle tracking error of the four motors

\subsection{Results of Mean Deviation Coupling Control}

In this paper, the control method is validated in motion (69). The displacement synchronization error of motor 1 with motor 2 is shown in Figure 12 and some other control methods including parallel control, master-slave control (Yang, Zuo \& Zhang, 2011) and relative coupling control (Cao, Wang \& Xiao-Yong, 2013) are compared with the improved mean deviation coupling in order to verify the collaborative performance. As it can be seen from Figure 12, the proposed mean deviation coupling control is characterized by lower synchronization error and better collaborative performance when compared to other traditional synchronous control methods. 


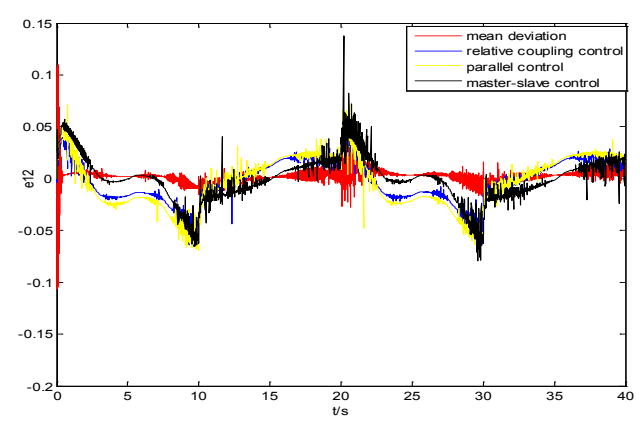

Figure 12. The displacement synchronization error of motor 1 with motor 2

\subsection{Results of the Effective Validation}

Simulation has been carried out using the Anybody software in order to prove the availability of the mechanism. The initial posture and the joint movement are shown in Figure 13. The muscle activity and muscle force during the motion of lateral flexion with and without extra assistance can be calculated through inverse dynamics analysis. Figure 14 illustrates the maximal muscle activity of the human body which reaches $39 \%$ in independent motion and $32 \%$ in assisted motion. Figure 15 displays the contrast force of the lumbar transverse process which drops down to $52 \mathrm{~N}$ after being power assisted. As Figure 16 illustrates, it becomes obvious that the force has been significantly reduced when compared to the force in motion without assistance for both the right and the left erector spinae. As it can be noticed, the designed exoskeleton can greatly relieve the lumbar pressure.

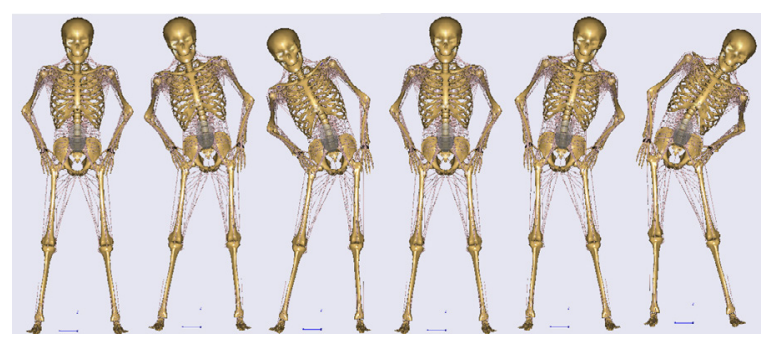

Figure 13. The movement process

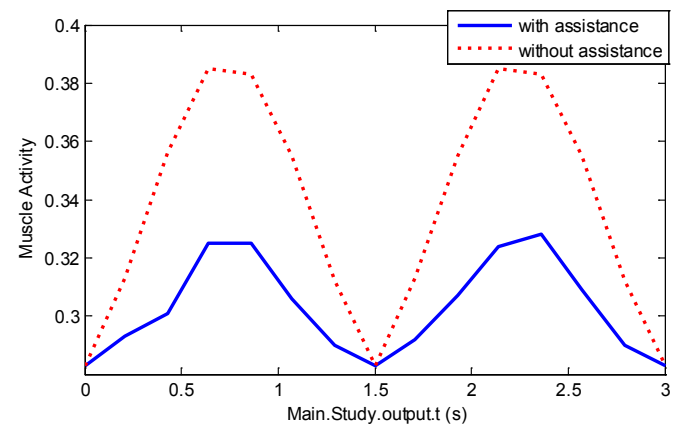

Figure 14. The maximal muscle activity

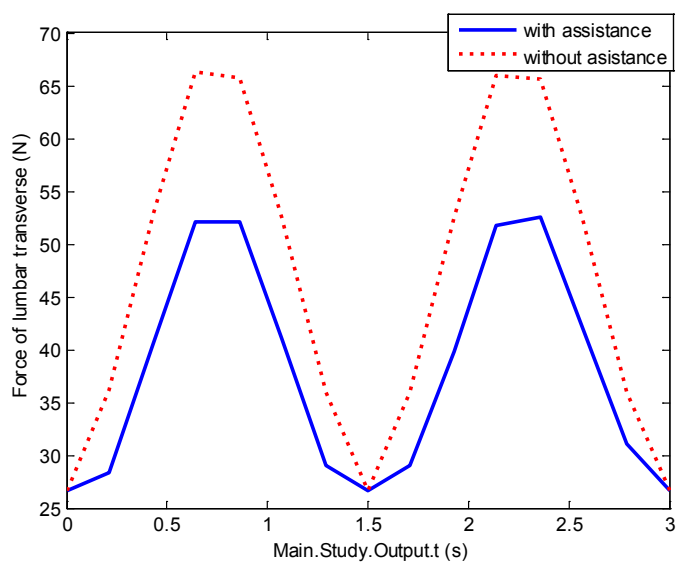

Figure 15. The force of the lumbar transverse process

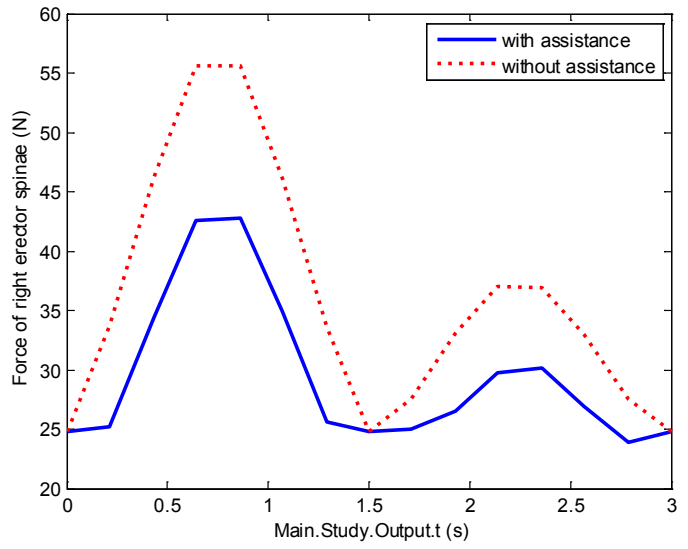

(a)Force of the right erector spinae

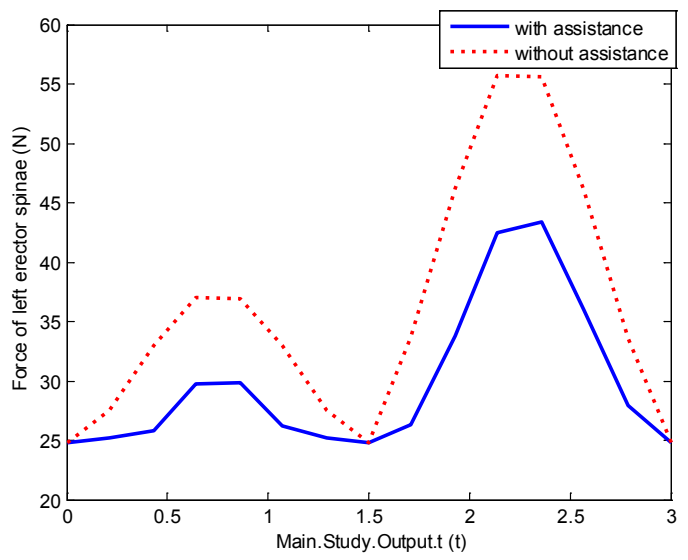

(b)Force of the left erector spinae

Figure 16. The force of erector spinae

\section{Conclusion and Future Work}

In this paper, a new wearable power assist device for lower back support is proposed to break through traditional limitation in structure which leads to the uniformity of the degree

https://www.sic.ici.ro 
of freedom and the influence on other joints. The degree of freedom of the mechanism is verified. The mathematical model of the device including the kinematics and the dynamics is also presented. In order to improve the security and practicality of the device, a modified LuGre model is established to describe the friction in the pushrod. Then, an adaptive compensation based on nonlinear observers is adopted to realize the motor angle tracking. An improved mean deviation coupling control method is proposed to realize the coordination work of the four motors. The simulation results show better performances when compared to other methods. Simulation has been carried out using the Anybody software in

\section{REFERENCES}

1. Abdoli-Eramaki, M., Stevenson, J. M., Reid, S. A. \& Bryant, T. J. (2007). Mathematical and empirical proof of principle for an onbody personal lift augmentation device (PLAD), Journal of Biomechanics, 40(8), 1694-1700.

2. Ahmed, S., Wang, H. \& Tian, Y. (2018). Fault Tolerant Control using Fractionalorder Terminal Sliding Mode Control for Robotic Manipulators, Studies in Informatics and Control, 27(1), 55-64. DOI: doi.org/10.24846/v27i1y201806

3. Ben-Israel, A. (1965). A Newton-Raphson method for the solution of systems of equations, Journal of Mathematical Analysis and Applications, 3(2), 94-98.

4. Canudas de Wit, C., Olsson, H., Astrom, K. J. \& Lischinsky, P. (1995). A new model for control of systems with friction, IEEE Transactions on Automatic Control, 40(3), 419-425.

5. Cao, C. P., Wang, B. \& Xiao-Yong, X. U. (2013). Research On Relative Coupling Control for Multi-motor Synchronization Based on Neural Network, Control Engineering of China, 20(3), 415-418.

6. Damsgaard, M., Rasmussen, J., Christensen, S. T. et al. (2006). Analysis of musculoskeletal systems in the AnyBody Modeling System, Simulation Modelling Practice and Theory, 14(8), 1100-1111.

7. Dehghani, M., Ahmadi, M., Khayatian, A. et al. (2008). Neural network solution order to prove the availability of the mechanism. A future work will focus upon a physical object based on this device. Other synchronous control methods will also be studied.

\section{Acknowledgments}

This work was partially supported by the National Natural Science Foundation of China (61773212), by International Science \& Technology Cooperation Program of China (2015DFA01710), by the Natural Science Foundation of Jiangsu Province (BK20170094), and by the 11th Jiangsu Province Six talent peaks of high level talents (2014_ZBZZ_005).

for forward kinematics problem of HEXA parallel robot. In Proceedings of the American Control Conference, 72 (pp. 63-70).

8. Hagen, K. B. \& Thune, O. (1998). Work incapacity from low back pain in the general population, Spine, 23(19), 2091-2095.

9. Hou, Y. L., Zhao, C. X. \& Fan, J. K. (2014). Kinematics and bionic design on spherical parallel hip joint mechanism of humanoid robot, Journal of Machine Design, 31(12), 25-30.

10. Imamura, Y., Tanaka, T., Suzuki, Y., Takizawa, K. \& Yamanaka, M. (2014). Analysis of trunk stabilization effect by passive power-assist device, Journal of Robotics and Mechatronics, 26(6), 791-798.

11. Inose, H., Mohri, S., Arakawa, H. et al. (2017). Semi-endoskeleton-type waist assist ABwear suit equipped with compressive force reduction mechanism. In IEEE International Conference on Robotics and Automation (ICRA), Singapore (pp. 6014-6019).

12. Inose, H., Mohri, S., Yamada, Y. et al. (2017). Development of a lightweight power-assist suit using pneumatic artificial muscles and balloon-amplification mechanism. In IEEE International Conference on Control, Automation, Robotics and Vision (pp. 1-6).

13. Kim, J. S., Jin, H. J. \& Park, J. H. (2015). Inverse kinematics and geometric singularity analysis of a 3-SPS/S redundant motion mechanism using conformal geometric 
algebra, Mechanism and Machine Theory, 90, 23-36.

14. Li, L., Sun, L. \& Zhang, S. (2016). Mean deviation coupling synchronous control for multiple motors via second-order adaptive sliding mode control, ISA Transactions, 62, 222-235.

15. Liu, J. F., Li, Y. W. \& Huang, Z. (2011). Mobility Analysis of Altmann Overconstrained Linkages by Modified Grübler-Kutzbach Criterion, Chinese Journal of Mechanical Engineering, 24(4), 638-646.

16. Luo, Z. \& Yu, Y. (2013). Wearable stoopingassist device in reducing risk of low back disorders during stooped work, IEEE International Conference on Mechatronics and Automation (pp. 230-236).

17. Maniadakis, N. \& Gray, A. (2000). The economic burden of back pain in the UK, Pain, 84(1), 95-103.

18. Verhoeven, R. \& Hiller, M. (2000). Estimating the Controllable Workspace of Tendon-Based Stewart Platforms,
Advances in Robot Kinematics, 277-284. Springer Netherlands.

19. Wang, Y. F., Wang, D. H. \& Chai, T. Y. (2011). Extraction and adaptation of fuzzy rules for friction modeling and control compensation, IEEE Transactions on Fuzzy Systems, 19(4), 682-93.

20. Wang, Y., Hu, J., Liu, L. et al. (2016). Integral backstepping control of servo actuators with LuGre model-based friction compensation. In IEEE International Conference on Aircraft Utility Systems (AUS) (pp. 984-990).

21. Yang, G. L., Zuo, G. Y. \& Zhang, C. C. (2011). Research of fuzzy PID control for master-slave mode water hydraulic parallel cylinders synchronous system, Chinese Hydraulics \& Pneumatics, 61(s1-2), 41-58.

22. Zhou, X., Zhao, B., Liu, W. et al. (2017). A compound scheme on parameters identification and adaptive compensation of nonlinear friction disturbance for the aerial inertially stabilized platform, ISA Transactions, 67, 293-305. 